\title{
Qualidade de vida após transplante penetrante de córnea
}

\author{
Assessment of quality oflife after penetrating keratoplasty
}

\author{
Denise Atique ${ }^{1}$ \\ Dario Grechi Goulart ${ }^{2}$ \\ Jonathan Clive Lake ${ }^{1}$ \\ Fabiana Amorim Lima ${ }^{1}$ \\ Sérgio Felberg ${ }^{1}$ \\ Maria Cristina Nishiwaki-Dantas ${ }^{3}$
}

${ }^{1}$ Médica(o) Assistente do Setor de Córnea e Moléstias Externas do Departamento de Oftalmologia da Santa Casa de São Paulo.

${ }^{2}$ Médico Assistente do Setor de Retina e Vítreo do Departamento de Oftalmologia da Santa Casa de São Paulo.

${ }^{3}$ Chefe do Setor de Córnea e Moléstias Externas do Departamento de Oftalmologia da Santa Casa de São Paulo.

Endereço para correspondência: Rua Professora Ruth Fonseca, 156 - Jundiaí (SP) CEP 13208-501.

E-mail: deatique@zaz.com.br

Recebido para publicação em 31.07.2001

Aceito para publicação em 12.12.2001

Nota Editorial: Pela análise deste trabalho e por sua anuência sobre a divulgação desta nota, agradecemos ao Dr. Elcio Hideo Sato.

\section{RESUMO}

Objetivo: Determinar a qualidade de vida dos pacientes submetidos a transplante penetrante de córnea por meio do teste VF-14 e correlacionála com a acuidade visual (AV) pós-operatória. Método: Cinqüenta e oito pacientes submetidos a transplante penetrante de córnea (mínimo de 1 ano após a operação) foram avaliados por meio do teste VF-14. Informações sobre o exame oftalmológico foram coletadas dos prontuários. A análise estatística utilizada foi a regressão linear e t de Student. Resultado: A média das pontuações do VF-14 foi 69,3. As maiores pontuações obtidas foram para atividades como cozinhar $(90,9)$, jogar (baralho, dominó) $(88,0)$ e realização de trabalhos manuais $(83,9)$. As menores, por sua vez, foram para dirigir veículos motorizados (à noite, 22,5 e durante o dia, 40,9). Os resultados foram estatisticamente significativos $(\mathrm{p}<0,01)$ e a regressão linear mostrou fraca correlação entre as pontuações obtidas e as AV pósoperatórias $(\mathrm{r}=0,41)$. Conclusão: A correlação entre a qualidade de vida e a acuidade visual pós-operatória mostrou-se fraca. Sugerimos modificações neste questionário, de modo a incluir atividades mais condizentes com o dia-a-dia de nossos pacientes.

Descritores: Transplante de córnea; Ceratoplastia; Qualidade de vida; Satisfação do paciente; Indicadores de saúde

\section{INTRODUÇÃO}

Transplante penetrante de córnea pode ser considerado cirurgia de sucesso, o qual é usualmente avaliado apenas pela acuidade visual final (AV), grau de transparência e ausência de rejeição ou recidiva de doença no botão corneal transplantado ${ }^{(1-2)}$. Não se avalia, entretanto, o grau de satisfação do paciente.

A qualidade de vida dos pacientes tem diferentes fatores a ser interpretados e, uma vez que saúde é definida não apenas como ausência de doença, mas também bem estar físico, mental e social, cada vez mais o fator "qualidade de vida" tem sido considerado ${ }^{(3-4)}$.

Desenvolvido nos Estados Unidos da América (EUA), o teste VF-14 mede a capacidade funcional (bem estar físico) relacionada à visão em pacientes submetidos à cirurgia de catarata. Baseia-se em 14 atividades executadas no dia a dia, que podem ser afetadas por doenças oculares ${ }^{(5)}$. Já o teste SF-36, tem a finalidade de avaliar a qualidade de vida relacionada à saúde geral do paciente ${ }^{(6-8)}$.

Ambos foram utilizados com o objetivo de determinar sua validade e eficácia em pacientes submetidos a transplante penetrante de córnea ${ }^{(9)}$. Outros autores estudaram a validade do VF-14 em pacientes que seriam submetidos a transplante (pré-operatório) ${ }^{(10)}$.

Não há, em nosso conhecimento, na literatura nacional, qualquer estudo 
sobre a qualidade de vida de pacientes após transplante penetrante de córnea. A diferença socioeconômica que há entre os EUA, país de origem da maioria destes estudos, e o Brasil, torna importante a utilização do questionário VF-14 em nossos pacientes.

\section{OBJETIVO}

O objetivo deste estudo foi determinar a qualidade de vida dos pacientes submetidos a transplante penetrante de córnea, pelo menos um ano após a cirurgia, por meio do questionário VF-14 e correlacioná-la com a AV pós-operatória final destes pacientes.

\section{MÉTODO}

Este estudo foi realizado no Setor de Córnea e Doenças Externas Oculares do Departamento de Oftalmologia da Santa Casa de Misericórdia de São Paulo, no período de fevereiro de 1999 a junho de 2000.

Os cinqüenta e oito pacientes submetidos a transplante penetrante de córnea foram avaliados um ano ou mais após a operação. A idade média foi de 49,5 $\pm 21,4$ anos (variou de 9 a 85 anos, sendo que apenas um paciente tinha nove anos e todos os outros tinham idade maior que dezoito anos); 23 $(39,7 \%)$ pacientes eram do sexo masculino e 35 (60,3\%), do feminino. Predominou a raça branca, com 45 pacientes $(77,5 \%)$. Ceratopatia bolhosa e ceratocone foram as principais indicações para o transplante penetrante de córnea (Tabela 1).

Foram excluídos pacientes que não tivessem condições de expressar-se devido à idade (crianças menores de sete anos) ou déficit mental e transplantes terapêuticos ou tectônicos de córnea.

Os pacientes, cientes do estudo científico do qual participaram, foram chamados por telefone e a entrevista, sempre realizada pelo mesmo médico, ocorreu pessoalmente.

O método de avaliação utilizado neste estudo foi o questio-

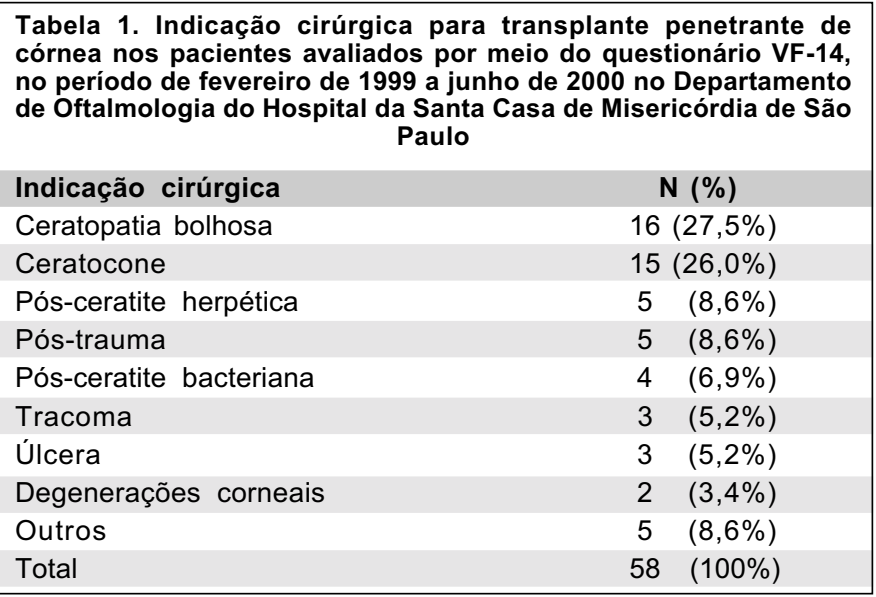

\begin{tabular}{|c|c|c|}
\hline \multicolumn{3}{|c|}{ Modelo do questionário utilizado } \\
\hline Atividade & Nota & Nota Final \\
\hline Ler jornal ou livros & & $\times 25=$ \\
\hline Reconhecimento de pessoas (de perto) & & $\times 25=$ \\
\hline Ver degraus & & $\times 25=$ \\
\hline Ler sinais de trânsito/ placas na rua & & $x 25=$ \\
\hline Trabalhos manuais & & $x 25=$ \\
\hline Escrever (cheques, recados) & & $\times 25=$ \\
\hline Jogar (cartas, dominó) & & $\times 25=$ \\
\hline Praticar esportes & & $\times 25=$ \\
\hline Cozinhar & & $\times 25=$ \\
\hline Assistir televisão & & $x 25=$ \\
\hline Dirigir durante o dia & & $\times 25=$ \\
\hline Dirigir à noite & & $\times 25=$ \\
\hline Ler letras miúdas & & $\times 25=$ \\
\hline Ler letras grandes & & $\times 25=$ \\
\hline
\end{tabular}

nário VF-14, de modo que 14 atividades dependentes da função visual foram avaliadas.

Os pacientes foram questionados quanto à dificuldade em executar cada uma das tarefas já mencionadas e responderam conferindo uma graduação às dificuldades: $0=$ impossível realizar; $1=$ dificuldade intensa; 2 = dificuldade moderada; 3 = dificuldade mínima; 4 = sem dificuldade.

Esta "nota" dada pelo paciente foi, então, multiplicada por 25 , para obter-se a pontuação, que variou de zero (pior índice de função visual) a 100 (melhor índice de função visual) $^{(5)}$.

Os métodos estatísticos utilizados foram de "regressão linear" e "T de student".

\section{RESULTADOS}

A acuidade visual, medida por meio da tabela de Snellen antes da cirurgia variou de percepção luminosa a 0,2 . Vinte e dois pacientes $(38,0 \%)$ apresentaram AV final (com correção óptica) melhor ou igual a $0,3^{(11)}$. A mediana foi 0,1 .

Das atividades avaliadas, houve maior dificuldade para dirigir veículos motorizados, tanto à noite como durante o dia, e para ler letras pequenas. Para dirigir à noite, dos dez pacientes que referiram tal prática, 7 apresentaram incapacidade para tal atividade e 1 mostrou dificuldade razoável, 1 teve pouca dificuldade e 1, nenhuma dificuldade. Para dirigir durante o dia, do total de 11 pacientes, 6 referiram grande dificuldade ou impossibilidade. Quanto à leitura de letras pequenas, 50,8\% $(n=28)$ foram incapazes ou apresentaram muita dificuldade.

As melhores pontuações foram obtidas para cozinhar (91\% mostrou mínima ou nenhuma dificuldade), jogar baralho ou dominó ( $90,5 \%$ não tiveram ou tiveram pouca dificuldade) e para realização de trabalhos manuais $(89,2 \%$ tiveram pouca ou nenhuma dificuldade). A tabela 2 mostra cada item avaliado.

A análise estatística demonstrou fraca correlação entre AV final e pontuação e o resultado foi estatisticamente significante $(r=0,41, p<0,01)$, conforme ilustrado no gráfico a seguir. 


\begin{tabular}{|c|c|c|c|c|c|c|c|c|}
\hline Atividade & $\begin{array}{l}\text { Impossível } \\
\text { realizar }\end{array}$ & $\begin{array}{l}\text { Dificuldade } \\
\text { intensa }\end{array}$ & $\begin{array}{l}\text { Dificuldade } \\
\text { moderada }\end{array}$ & $\begin{array}{l}\text { Dificuldade } \\
\text { mínima }\end{array}$ & $\begin{array}{c}\text { Sem } \\
\text { dificuldade }\end{array}$ & Total & $\begin{array}{l}\text { Não se } \\
\text { aplicou }\end{array}$ & Média \\
\hline Dirigir à noite & $7(70 \%)$ & 0 & $1(10,0 \%)$ & $1(10,0 \%)$ & $1(10,0 \%)$ & $10(100 \%)$ & $48(82,7 \%)$ & 22,5 \\
\hline Dirigir de dia & $5(45,4 \%)$ & $1 \quad(9,1 \%)$ & $1 \quad(9,1 \%)$ & $1 \quad(9,1 \%)$ & $3(27,3 \%)$ & $11(100 \%)$ & $47(81,0 \%)$ & 40,9 \\
\hline Letras pequenas & $19(34,5 \%)$ & $9(16,3 \%)$ & $9(16,3 \%)$ & $5 \quad(9,1 \%)$ & $13(23,8 \%)$ & $55(100 \%)$ & $3 \quad(5,2 \%)$ & 42,7 \\
\hline Escadas & 0 & $7(13,1 \%)$ & $10(18,9 \%)$ & $15(28,3 \%)$ & $21(39,7 \%)$ & $53(100 \%)$ & $5 \quad(8,7 \%)$ & 73,5 \\
\hline Sinais de trânsito & 0 & $14(24,5 \%)$ & $10(17,5 \%)$ & $15(26,4 \%)$ & $18(31,6 \%)$ & $57(100 \%)$ & $1 \quad(1,7 \%)$ & 66,2 \\
\hline Escrever & 0 & $6(13,7 \%)$ & $6(13,7 \%)$ & $8(18,1 \%)$ & $24(54,4 \%)$ & $44(100 \%)$ & $14(24,1 \%)$ & 78,4 \\
\hline Jogar & 0 & $1 \quad(4,7 \%)$ & $1 \quad(4,7 \%)$ & $5(23,8 \%)$ & $14(66,7 \%)$ & $21(100 \%)$ & $37(63,7 \%)$ & 88,0 \\
\hline Esportes & 0 & $3(16,6 \%)$ & $2(11,1 \%)$ & $2(11,1 \%)$ & $11(61,2 \%)$ & $18(100 \%)$ & $40(69,0 \%)$ & 79,1 \\
\hline TV & 0 & $6(12,3 \%)$ & $10(20,5 \%)$ & $11(22,5 \%)$ & $22(44,9 \%)$ & $49(100 \%)$ & $9(15,6 \%)$ & 75,0 \\
\hline
\end{tabular}

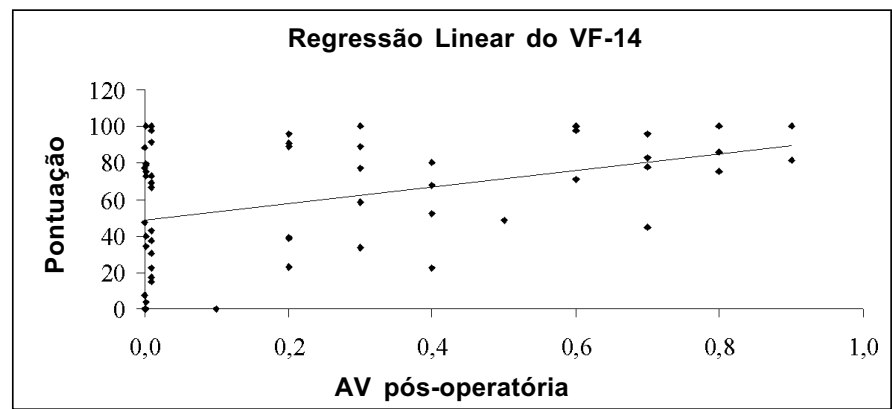

Gráfico 1 - Gráfico da regressão linear da correlação entre AV final e pontuação média obtida pelo questionário VF-14, para cada paciente submetido a transplante penetrante de córnea, no período de fevereiro de 1999 a junho de 2000, no Departamento de Oftalmologia do Hospital da Santa Casa de Misericórdia de São Paulo

\section{COMENTÁRIOS}

O questionário VF-14 mostrou- se válido para se avaliar a qualidade de vida de pacientes após transplante penetrante de córnea. Em nosso estudo, a pontuação média foi de 69,3, semelhante à encontrada $(68,3)$ em Barcelona, para pacientes após cirurgia de catarata; entretanto, foi inferior à média obtida para transplante penetrante de córnea (77) e para catarata, nos $\operatorname{EUA}(75,5)^{(5,9)}$.

A cirurgia de catarata é um procedimento tecnicamente mais simples, menos traumático e seus resultados, geralmente muito bons, aparecem até mesmo horas após a operação. $\mathrm{O}$ transplante de córnea, por sua vez, tem período de recuperação prolongado, com seguimento mínimo de 6 meses a um ano, e os resultados visuais geralmente começam a aparecer a partir de quatro meses após a cirurgia. Além disso, complicações após o transplante são mais freqüentes, como rejeição, glaucoma e astigmatismo ${ }^{(5,12-13)}$.

Portanto, esperaríamos que a pontuação do teste VF-14 fosse maior após cirurgia de catarata que após transplante de córnea. Entretanto, os estudos americanos para ambas as cirurgias obtiveram pontuações semelhantes $(75,5$ e 77,0 , respectivamente, nos estudos para catarata e transplante de córnea) $)^{(5,9)}$.

Em nosso estudo, da mesma forma que nos estudos americanos, o sexo feminino foi mais freqüentemente acometido $(60,3 \%)$, assim como a raça branca. Entretanto, nossos pacientes eram mais jovens (idade média de 49,5 anos) e as indicações pré-operatórias não apresentavam a mesma distribuição.

No estudo americano ${ }^{(9)}$, ceratopatia bolhosa do afácico, pseudofacia e distrofia de Fuchs, que geralmente acometem indivíduos mais idosos, foram responsáveis por dois terços das indicações para cirurgia, enquanto, em nosso estudo, esse grupo foi responsável por apenas um terço das indicações. Ceratocone foi, em nossos pacientes $(26,0 \%)$, mais freqüente que no estudo americano $(13,0 \%)^{(9)}$. Portanto, a idade média inferior era esperada para nossos pacientes.

Obtivemos, além disso, maior número de indicações para casos pós-infecção e pós-traumatismo, que geralmente acometem indivíduos mais jovens, com córneas mais vascularizadas e portanto com pior prognóstico para transplante de córnea ${ }^{(13)}$.

Outra dificuldade encontrada foi o fato de que muitas das atividades exploradas pelo questionário não fazem parte do dia a dia da nossa população, como dirigir veículos motorizados (a maior parte deles não possui carro e um paciente tinha apenas nove anos de idade), praticar esportes (alguns são idosos ou o tempo dedicado ao trabalho não permitiu) e ler e escrever (muitos são semi-analfabetos ou analfabetos).

A análise estatística revelou que existe fraca correlação entre a média das pontuações obtidas para cada paciente e suas respectivas $\mathrm{AV}$ finais com correção óptica $(\mathrm{p}<0,01$; $\mathrm{r}=0,41$ ), isto é, nem todo paciente que obteve pontuação alta tinha AV compatível e vice-versa.

A pontuação final média de 69,3 em nosso estudo foi inferior à de outros trabalhos $(77)^{(9)}$. Possíveis explicações 
seriam o fato de que a mediana da $\mathrm{AV}$ em nosso estudo foi inferior $(0,1)$, muitos de nossos pacientes apresentavam pior resultado do transplante de córnea (pós-infecção e trauma) e a idade média foi menor. Portanto, pacientes mais jovens têm provavelmente maior expectativa com relação ao resultado após transplante de córnea (pontuação final menor) e as AV mais baixas também justificariam a menor pontuação.

\section{CONCLUSÃO}

Existe fraca correlação entre a pontuação final média $(69,3)$ e a AV final, isto é, pacientes com AV semelhantes nem sempre compartilham o mesmo grau de satisfação após o transplante de córnea.

Sugerimos modificação deste questionário, de modo a incluir atividades mais condizentes com a realidade de nossos pacientes.

\section{ABSTRACT}

Purpose: To determine the quality of life in patients after penetrating keratoplasty using the VF-14 test and correlating it with final visual acuity. Methods: A total of 58 penetrating keratoplasty operated patients (1 year after surgery) were evaluated by the VF-14 test. Ophthalmological examination data were collected from their charts. Student's t test and linear regression were applied. Results: The VF-14 ratio was 69.3. Best scores were obtained in activities such as cooking (90.9), card games (88.0), fine handworks (83.9). On the contrary, worst scores were found for car driving (at night, 22.5; and during the day, 40.9). Results were statistically significant $(p<0.01)$ and linear regression showed weak correlation between scores and final visual acuity $(r=0.41)$. Conclusion: We observed a weak correlation between the quality of life and final visual acuity and suggest changes in this questionnaire including activities that are more adequate for our patients.

Keywords: Corneal transplantation; Keratoplasty; Quality of life; Patient satisfaction; Health status indicators

\section{REFERÊNCIAS}

1. Borderie VM, Lopes M, Vedie F, Laroche L. ABO antigen blood-group compatibility in corneal transplantation. Cornea 1997;16:1-6.

2. The Colaborative Corneal Transplantation Studies Research Group. Effectiveness of histocompatibility matching in high-risk corneal transplantation. Arch Ophthalmol 1992;110:1392-403.

3. Gill TM, Feinstein AR. A critical appraisal of the quality of life. JAMA 1994;272:619-26.

4. Testa MA, Simonson DC. Assessment of quality of life outcomes. N Engl Med J 1996;334:835-40.

5. Alonso J, Espallargues M, Andersen TF, Cassard SD, Dunn E, BernthPetersen $\mathrm{P}$ et al. International applicability of the VF-14. An index of visual function in patients with cataracts. Ophthalmology 1997;104:799-807.

6. Lee PP, Spritzer K, Hays RD. The impact of blurred vision on functioning and well-being. Ophthalmology 1997;104:390-6.

7. Parrish RK, Gedde SJ, Scott IU, Feuer WJ, Schiffman JC, Mangione CM et al. Visual function and quality of life among patients with glaucoma. Arch Ophthalmol 1997;115:1447-55.

8. Ware JE, Sherbourne CD. The MOS 36-item short-form health survey (SF36). I. Conceptual framework and item selection. Med Care 1992;30:473-83.

9. Musch DC, Farjo AA, Meyer RF, Waldo MN, Janz NK. Assessment of health-related quality of life after corneal transplantation. Am J Ophthalmol 1997;24:1-8.

10. Boisjoly H, Gresset J, Fontaine N, Charest M, Brunette I, LeFrançois M et al. The VF-14 index of functional visual impairment in candidates for a corneal graft. Am J Ophthalmol 1999;128:38-44.

11. Holladay JT. Proper method for calculating average virtual acuity. J Refract Surg 1997;13:388-91.

12. Dantas PEC, Kara-José N. Tratamento das complicações no período pósoperatório de transplante de córnea-guia de sobrevivência para o oftalmologista geral. Sinopse Oftalmol 1999;2:36-40.

13. Williams KA, Ash JK, Pararajasegaram P, Harris S, Coster DJ. Long-term outcome after corneal transplantation. Ophthalmology 1991;98:651-7.

\title{
ABO ELETRÔNICO
}

\author{
Novo site
}

Acesso: http://www.abonet.com.br 